\title{
TRANSIENT ANALYSIS OF A FLUID QUEUE DRIVEN \\ BY A BIRTH AND DEATH PROCESS SUGGESTED \\ BY A CHAIN SEQUENCE
}

\author{
P. R. PARTHASARATHY, B. SERICOLA, AND K. V. VIJAYASHREE
}

Received 31 March 2004 and in revised form 30 August 2004

We analyse the transient behaviour of a fluid queue driven by a birth and death process (BDP) whose birth and death rates are suggested by a chain sequence. For the BDP suggested by a chain sequence, the stationary probabilities do not exist and hence the stationary buffer content distribution for fluid queues driven by such BDP does not exist. However, their transient distribution is obtained in a simple closed form by two different approaches: the first is the continued fraction approach and the second is an approach in terms of recurrence relation by an analysis similar to that of Sericola (1998). The probability for the buffer content to be empty at an arbitrary time is also studied. The variations in this performance measure are revealed in the form of graphs. Numerical illustrations are included.

\section{Introduction}

Fluid buffer models are natural for problems involving continuous flow, for example, control of dams, virtual waiting time in $G / G / 1$ queue, and so forth. In addition, fluid models are often useful as approximate models for certain queueing and inventory systems where the flow consists of discrete entities, but the behaviour of individuals is not important to identify the performance analysis (Kulkarni [3]). A stochastic fluid flow model describes the behaviour of a fluid level in a storage device. Such models are used in ATM to evaluate the performance of fast packet switching and in the manufacturing systems for the performance of producers and consumers coupled by a buffer.

Hence fluid queues modulated by a birth and death process provide a good approximation for modelling the traffic in telecommunication networks. However in most practical situations, the state-dependent birth and death rates prove to be more realistic.

Steady-state behaviour of Markov-driven fluid queues have been extensively studied in the literature (Virtamo and Norros [14], Adan and Resing [1], Parthasarathy et al. [7], Parthasarathy and Vijayashree [5, 6], Barbot and Sericola [2], van Doorn and Scheinhardt [13], Sericola [10]). Steady state analysis gives us important information on the congestion of the statistical multiplexer, but it is not sufficient, for example, for controlling the congestion. The transient analysis will be of critical value in understanding the dynamical 
behaviour of the statistical multiplexer in controlling the congestion and in studies relating to the rate of convergence to steady state. The problem is motivated by the need to comprehend better the performance of fast packet switching in asynchronous transfer mode (ATM), which will be adopted in broadband integrated service digital network (BISDN).

The transient analysis of stochastic fluid models presents a host of new challenges and opportunities to network designers and performance analyst. The solutions are either obtained implicitly (Simonian and Virtamo [11]) or obtained in the Laplace domain and inverted numerically (Tanaka et al. [12], Ren and Kobayashi [8], Sericola [9]).

In all the above-mentioned literature, the authors discussed the transient analysis of the fluid models subject to satisfying the stability condition of the process. Here we consider a fluid queue driven by an infinite-state BDP whose birth and death rates are suggested by a chain sequence. The stationary solution for the background BDP suggested by a chain sequence does not exist and hence the stationary distribution for fluid queue driven by such BDPs also does not exist. However their transient probabilities yield a simple closed form solution.

In this paper, we obtain the transient solution in closed form of a fluid queue driven by a birth and death process on an infinite-state space whose birth and death rates are suggested by a chain sequence. The probability with which the buffer content becomes empty at an arbitrary time is also determined. Numerical illustrations are added to capture the variations in the behaviour of this performance measure against time.

\section{Model description}

Consider a fluid queue driven by a birth and death process, $\{X(t), t \geq 0\}$ with rates suggested by a chain sequence, viz, the birth and death parameters satisfy

$$
\lambda_{n}+\mu_{n}=1, \quad \lambda_{n-1} \mu_{n}=\beta, \quad \text { i.e., }\left(1-\mu_{n-1}\right) \mu_{n}=\beta, \quad n=1,2,3, \ldots,
$$

with $\lambda_{0}=1$ and $\mu_{0}=0$ so that $\left\{\mu_{n}\right\}$ is the minimal parameter sequence for the constant term chain sequence $\{\beta, \beta, \beta, \ldots\}, 0<\beta \leq 1 / 4$, so that $\lambda_{n}$ and $\mu_{n}$ are positive, given by

$$
\begin{aligned}
& \lambda_{n}=\frac{\alpha U_{n+1}(1 / \alpha)}{2 U_{n}(1 / \alpha)}, \quad n=1,2,3, \ldots, \\
& \mu_{n}=\frac{\alpha U_{n-1}(1 / \alpha)}{2 U_{n}(1 / \alpha)}, \quad n=1,2,3, \ldots,
\end{aligned}
$$

where $U_{n}(\cdot)$ is the Chebyshev polynomial of second kind of order $n$ and $\alpha=2 \sqrt{\beta}$. Note that

$$
\mu_{1} \mu_{2} \cdots \mu_{j}=\left(\frac{\alpha}{2}\right)^{j} \frac{1}{U_{j}(1 / \alpha)}=\frac{(\sqrt{\beta})^{j}}{U_{j}(1 / 2 \sqrt{\beta})}
$$


The transition probabilities for the process $\{X(t), t \geq 0\}$, whose birth and death rates are governed by (2.1), with $X(0)=0$, are

$$
P_{n}(t)=2(n+1) U_{n}\left(\frac{1}{\alpha}\right) \frac{e^{-t} I_{n+1}(\alpha t)}{\alpha t}
$$

(Lenin and Parthasarathy [4].)

It can easily be shown that the sequence $\left\{\lambda_{n}\right\}$ is decreasing with $n$ and tends towards $(1+\sqrt{1-4 \beta}) / 2$, so that it could represent a queue with discouraged arrivals. The sequence $\left\{\mu_{n}\right\}$ is thus increasing with $n$ towards $(1-\sqrt{1-4 \beta}) / 2$, which means that the service rate of the queue can be dynamically adapted in the function of the number of customer in the queue, until a fixed limit. This kind of model is mathematically interesting because it is indeed rare and has closed-form solution.

If $C(t)$ denotes the content of the buffer at time $t$, the 2-dimensional process $\{(X(t), C(t)), t \geq 0\}$ constitutes a Markov process. When the process $X(t)$ is positive, the fluid level in the buffer increases at a constant rate $r>0$ and when $X(t)=0$, the fluid level in the buffer decreases at a constant rate $r_{0}<0$. We suppose that $X(0)=0$ and $C(0)=0$. Fluid models of this type find application in the field of telecommunication for modelling the network traffic and in the approximation of discrete stochastic queueing networks. For practical design and performance evaluation, it is essential to obtain information about the buffer occupancy distribution.

If $F_{j}(t, x) \equiv P(X(t)=j, C(t) \leq x), j \in \mathscr{S}, t, x \geq 0$, the Kolmogorov forward equations for the Markov process $\{X(t), C(t)\}$ are given by

$$
\begin{gathered}
\frac{\partial F_{0}(t, x)}{\partial t}=-r_{0} \frac{\partial F_{0}(t, x)}{\partial x}-F_{0}(t, x)+\mu_{1} F_{1}(t, x), \\
\frac{\partial F_{j}(t, x)}{\partial t}=-r \frac{\partial F_{j}(t, x)}{\partial x}+\lambda_{j-1} F_{j-1}(t, x)-F_{j}(t, x)+\mu_{j+1} F_{j+1}(t, x), \quad j \in \mathscr{S} \backslash\{0\}, t, x \geq 0,
\end{gathered}
$$

subject to the initial condition

$$
F_{0}(0, x)=1, \quad F_{j}(0, x)=0 \quad \text { for } j=1,2,3, \ldots
$$

and boundary condition

$$
F_{j}(t, 0)=q_{j}(t) \quad \text { for } j=0,1,2, \ldots
$$

Here $q_{j}(t)$ represents the probability that at time $t$ the buffer is empty and the state of the background Markov process is $j$. The content of the buffer decreases and thereby becomes empty only when the net input rate of the fluid into the buffer is negative. Therefore, when the buffer becomes empty at any time $t$, the background process should necessarily be in state zero corresponding to which the effective input rate is $r_{0}<0$. Hence we have $q_{j}(t)=0$ for $j=1,2,3, \ldots$ as $r>0$ when $X(t)=j$ for $j=1,2,3, \ldots$. 
The transient distribution of the buffer content is given by

$$
\operatorname{Pr}(C(t)>x)=1-\sum_{j=0}^{\infty} F_{j}(t, x)
$$

In this sequel let $F_{j}^{*}(s, x)$ and $F_{j}^{* *}(s, w)$ denote the single Laplace transform (with respect to $t$ ) and double Laplace transform (with respect to $t$ and $x$ ) of $F_{j}(t, x)$, respectively.

\section{Transient solution}

The expression for the joint distribution of the buffer content of the fluid queue model under consideration using an approach similar to Sericola [9] is given by

$$
F_{i}(t, x)=\sum_{n=0}^{\infty} e^{-t} \frac{t^{n}}{n !} \sum_{k=0}^{n}\left(\begin{array}{l}
n \\
k
\end{array}\right)\left(\frac{x}{r t}\right)^{k}\left(1-\frac{x}{r t}\right)^{n-k} b_{i}(n, k), \quad i=0,1,2, \ldots
$$

for every $t \geq 0$ and $x \in[0, r t)$ where the coefficients $b_{i}(n, k)$ are given by the following recursive expressions.

(i) For $i=0$,

$$
\begin{gathered}
b_{0}(n, n)= \begin{cases}\frac{1}{k+1}\left(\begin{array}{c}
2 k \\
k
\end{array}\right) \beta^{k} & \text { if } n=2 k, \\
0 & \text { if } n=2 k+1,\end{cases} \\
b_{0}(n, k)=\frac{-r_{0}}{r-r_{0}} b_{0}(n, k+1)+\frac{r \beta}{r-r_{0}} b_{1}(n-1, k) \quad \text { for } n \geq 1,0 \leq k \leq n-1 .
\end{gathered}
$$

(ii) For $i \geq 1$,

$$
\begin{aligned}
& b_{i}(n, 0)=0 \quad \text { for } n \geq 0, \\
& b_{i}(n, k)=\lambda_{i-1} b_{i-1}(n-1, k-1)+\mu_{i+1} b_{i+1}(n-1, k-1) \quad \text { for } n \geq 1,1 \leq k \leq n \text {. }
\end{aligned}
$$

From (3.1), the probability that the buffer is empty at time $t$ is given by

$$
F_{0}(t, 0)=q_{0}(t)=e^{-t} \sum_{n=0}^{\infty} \frac{t^{n}}{n !} b_{0}(n, 0)
$$

where $b_{0}(n, 0)$ for all $n \geq 1$ are obtained from the recurrence relations (3.2) and (3.3). The following theorem presents an alternate formula for the evaluation of $b_{0}(n, 0)$.

Theorem 3.1. For all $n \geq 1$,

$$
\begin{aligned}
b_{0}(n, 0)= & \frac{r \beta}{r-r_{0}} \sum_{i=1}^{n-1}\left(\frac{-r_{0}}{r-r_{0}}\right)^{i\lfloor(i-1) / 2\rfloor} \sum_{l=0}^{2 l}\left(\begin{array}{c}
l \\
l
\end{array}\right) \frac{\beta^{l}}{l+1} b_{0}(n-2 l-2, i-2 l-1) \\
& +\left(\frac{-r_{0}}{r-r_{0}}\right)^{n} b_{0}(n, n) .
\end{aligned}
$$


Proof. The following propositions and lemma present a simplified formula for evaluating the various terms involved in the determination of $b_{0}(n, 0)$ thereby reducing the computational complexity.

Proposition 3.2. For all $n \geq 1,0 \leq k \leq n-1$,

$$
b_{0}(n, k)-\left(\frac{-r_{0}}{r-r_{0}}\right)^{n-k} b_{0}(n, n)=\left(\frac{r \beta}{r-r_{0}}\right) \sum_{i=k}^{n-1}\left(\frac{-r_{0}}{r-r_{0}}\right)^{i-k} b_{1}(n-1, i) .
$$

Proof. Recall (3.2),

$$
b_{0}(n, k)=\frac{-r_{0}}{r-r_{0}} b_{0}(n, k+1)+\frac{r \beta}{r-r_{0}} b_{1}(n-1, k) .
$$

Multiplying the above equation by $\left(-r_{0} /\left(r-r_{0}\right)\right)^{i-k}$ and summing over all $i$ from $k$ to $n-1$, we get

$$
\begin{gathered}
\sum_{i=k}^{n-1}\left(\frac{-r_{0}}{r-r_{0}}\right)^{i-k} b_{0}(n, i)-\sum_{i=k}^{n-1}\left(\frac{-r_{0}}{r-r_{0}}\right)^{i-k+1} b_{0}(n, i+1) \\
=\left(\frac{r \beta}{r-r_{0}}\right) \sum_{i=k}^{n-1}\left(\frac{-r_{0}}{r-r_{0}}\right)^{i-k} b_{1}(n-1, i) .
\end{gathered}
$$

Hence we have

$$
b_{0}(n, k)-\left(\frac{-r_{0}}{r-r_{0}}\right)^{n-k} b_{0}(n, n)=\left(\frac{r \beta}{r-r_{0}}\right) \sum_{i=k}^{n-1}\left(\frac{-r_{0}}{r-r_{0}}\right)^{i-k} b_{1}(n-1, i) .
$$

Lemma 3.3. For $i \geq 1, b_{i}(n, k)=0$ for $0 \leq n<i$ and

$$
b_{i}(n, k)= \begin{cases}0 & \text { if } 0 \leq k<i, \\ (\sqrt{\beta})^{i} U_{i}\left(\frac{1}{2 \sqrt{\beta}}\right)^{\lfloor(k-i) / 2\rfloor} \sum_{l=0} s(i, l) \beta^{l} b_{0}(n-2 l-i, k-2 l-i) & \text { if } i \leq k \leq n, \\ 0 & \text { if } k>n,\end{cases}
$$

where the numbers $s(i, l)$ are referred to as the ballot numbers given by

$$
s(i, l)=i \frac{(2 l+i-1) !}{l !(l+i) !} .
$$

Proof. Recall (3.3),

$$
b_{i}(n, k)=\lambda_{i-1} b_{i-1}(n-1, k-1)+\mu_{i+1} b_{i+1}(n-1, k-1) \quad \text { for } n \geq 1,1 \leq k \leq n .
$$

For all $n \geq 1,1 \leq k \leq n$, define

$$
\begin{gathered}
B_{0}(n, k)=b_{0}(n, k), \\
B_{i}(n, k)=\left(\mu_{1} \mu_{2} \cdots \mu_{i}\right) b_{i}(n, k), \quad i \geq 1 .
\end{gathered}
$$


148 Fluid queue with chain sequence

Then (3.3) becomes

$$
B_{i}(n, k)=\lambda_{i-1} \mu_{i} B_{i-1}(n-1, k-1)+B_{i+1}(n-1, k-1) .
$$

From (2.1), $\lambda_{i-1} \mu_{i}=\beta$, hence we have

$$
B_{i}(n, k)=\beta B_{i-1}(n-1, k-1)+B_{i+1}(n-1, k-1) .
$$

Now, define

$$
H_{i}(n, v)=\sum_{k=0}^{n} v^{k} B_{i}(n, k)
$$

then $(3.15)$ reduces to

$$
H_{i}(n, v)=v \beta H_{i-1}(n-1, v)+v H_{i+1}(n-1, v) \quad i \geq 1, n \geq 1 .
$$

Again define

$$
\phi_{i}(u, v)=\sum_{n=0}^{\infty} \frac{u^{n} H_{i}(n, v)}{n !}
$$

then (3.17) reduces to

$$
\phi_{i}^{\prime}(u, v)=v \beta \phi_{i-1}(u, v)+v \phi_{i+1}(u, v) \quad \text { for } i \geq 1 .
$$

Laplace transform of the above equation with respect to $u$ yields

$$
z \phi_{i}^{*}(z, v)=v \beta \phi_{i-1}^{*}(z, v)+v \phi_{i+1}^{*}(z, v) .
$$

Writing in the form of continued fractions, we get

$$
\frac{\phi_{i}^{*}(z, v)}{\phi_{i-1}^{*}(z, v)}=\frac{v \beta}{z-v\left(\phi_{i+1}^{*}(z, v) / \phi_{i}^{*}(z, v)\right)}=\frac{v \beta}{z-} \frac{v^{2} \beta}{z-} \frac{v^{2} \beta}{z-} \cdots .
$$

Solving the above continued fractions, we get

$$
\begin{aligned}
& \frac{\phi_{i}^{*}(z, v)}{\phi_{i-1}^{*}(z, v)}=\frac{z-\sqrt{z^{2}-4 v^{2} \beta}}{2 v}, \quad i=1,2,3, \ldots, \\
& \frac{\phi_{i}^{*}(z, v)}{\phi_{i-1}^{*}(z, v)}=\frac{v \beta}{z}\left(\frac{1-\sqrt{1-4\left(v^{2} \beta / z^{2}\right)}}{2\left(v^{2} \beta / z^{2}\right)}\right)=\frac{v \beta}{z} C\left(\frac{v^{2} \beta}{z^{2}}\right) .
\end{aligned}
$$

Before we proceed further, we give a brief discussion on the function $C(z)$ below. Let $C(z)$ be the complex function defined by

$$
C(z)=\frac{1-\sqrt{1-4 z}}{2 z}
$$


For $|z| \leq 1 / 4$, we have

$$
C(z)=\sum_{n=0}^{\infty} c_{n} z^{n},
$$

where the numbers $c_{n}$ are referred to as the Catalan number given by

$$
c_{n}=\left(\begin{array}{c}
2 n \\
n
\end{array}\right) \frac{1}{n+1} .
$$

More generally, for $k \geq 1$ and $|z| \leq 1 / 4$, we have

$$
C^{k}(z)=\sum_{n=0}^{\infty} s(k, n) z^{n}
$$

where the numbers $s(k, n)$ are given by (3.11).

Continuing our discussion from (3.22), we easily get, for $i \geq 1$ and $\left|v \beta / z^{2}\right|<1 / 4$,

$$
\begin{aligned}
\phi_{i}^{*}(z, v) & =\frac{v \beta}{z} C\left(\frac{v^{2} \beta}{z^{2}}\right) \phi_{i-1}^{*}(z, v) \\
& =\frac{v^{i} \beta^{i}}{z^{i}} C^{i}\left(\frac{v^{2} \beta}{z^{2}}\right) \phi_{0}^{*}(z, v)=\frac{v^{i} \beta^{i}}{z^{i}} \sum_{l=0}^{\infty} s(i, l)\left(\frac{v^{2} \beta}{z^{2}}\right)^{l} \phi_{0}^{*}(z, v) .
\end{aligned}
$$

We thus have, for $i \geq 1$ and $\left|v \beta / z^{2}\right|<1 / 4$,

$$
\begin{aligned}
\sum_{n=0}^{\infty} \frac{H_{i}(n, v)}{z^{n+1}} & =\frac{v^{i} \beta^{i}}{z^{i}} \sum_{l=0}^{\infty} s(i, l)\left(\frac{v^{2} \beta}{z^{2}}\right)^{l} \sum_{n=0}^{\infty} \frac{H_{0}(n, v)}{z^{n+1}} \\
& =\beta^{i} \sum_{l=0}^{\infty} s(i, l) \beta^{l} v^{2 l+i} \sum_{n=0}^{\infty} \frac{H_{0}(n, v)}{z^{2 l+i+n+1}} \\
& =\beta^{i} \sum_{l=0}^{\infty} s(i, l) \beta^{l} v^{2 l+i} \sum_{n=2 l+i}^{\infty} \frac{H_{0}(n-2 l-i, v)}{z^{n+1}} \\
& =\beta^{i} \sum_{n=i}^{\infty} \frac{1}{z^{n+1}} \sum_{l=0}^{\lfloor(n-i) / 2\rfloor} s(i, l) \beta^{l} v^{2 l+i} H_{0}(n-2 l-i, v),
\end{aligned}
$$

where the last equality is obtained by exchanging the order of summations. This leads, for $i \geq 1$, to the following expression of $H_{i}(n, v)$ :

$$
H_{i}(n, v)= \begin{cases}0 & \text { if } 0 \leq n<i, \\ \beta^{i} \sum_{l=0}^{\lfloor(n-i) / 2\rfloor} s(i, l) \beta^{l} v^{2 l+i} H_{0}(n-2 l-i, v) & \text { if } n \geq i .\end{cases}
$$

This means, in particular, that $b_{i}(n, k)=0$ for $i \geq 1$ and $0 \leq n<i$. 
We consider now the case where $i \geq 1$ and $n \geq i$. By definition of $H_{i}(n, v)$, we have

$$
\begin{aligned}
\sum_{k=0}^{n} v^{k} B_{i}(n, k) & =\beta^{i} \sum_{l=0}^{\lfloor(n-i) / 2\rfloor} s(i, l) \beta^{l} v^{2 l+i} \sum_{m=0}^{n-2 l-i} v^{m} B_{0}(n-2 l-i, m) \\
& =\beta^{i} \sum_{l=0}^{\lfloor(n-i) / 2\rfloor} s(i, l) \beta^{l} \sum_{m=0}^{n-2 l-i} v^{m+2 l+i} B_{0}(n-2 l-i, m) \\
& =\beta^{i} \sum_{l=0}^{\lfloor(n-i) / 2\rfloor} s(i, l) \beta^{l} \sum_{k=2 l+i}^{n} v^{k} B_{0}(n-2 l-i, k-2 l-i) \\
& =\beta^{i} \sum_{k=i}^{n} v^{k} \sum_{l=0}^{\lfloor(k-i) / 2\rfloor} s(i, l) \beta^{l} b_{0}(n-2 l-i, k-2 l-i),
\end{aligned}
$$

where the last equality is obtained by exchanging the order of summations. This leads, for $i \geq 1$ and $n \geq i$, to the following expression of $B_{i}(n, k)$ :

$$
B_{i}(n, k)= \begin{cases}0 & \text { if } 0 \leq k<i, \\ \beta^{i} \sum_{l=0}^{\lfloor(k-i) / 2\rfloor} s(i, l) \beta^{l} B_{0}(n-2 l-i, k-l-i) & \text { if } i \leq k \leq n \\ 0 & \text { if } k>n .\end{cases}
$$

Using the transformation (3.13) and also from (2.3), we get

$$
b_{i}(n, k)= \begin{cases}0 & \text { if } 0 \leq k<i \\ (\sqrt{\beta})^{i} U_{i}\left(\frac{1}{2 \sqrt{\beta}}\right) \sum_{l=0}^{\lfloor(k-i) / 2\rfloor} s(i, l) \beta^{l} b_{0}(n-2 l-i, k-l-i) & \text { if } i \leq k \leq n \\ 0 & \text { if } k>n\end{cases}
$$

In particular, for $i=1$, the ballot numbers $s(1, l)$ are the Catalan number $c_{l}$. Thus,

$$
b_{1}(n, k)= \begin{cases}0 & \text { if } k=0, \\
\sum_{l=0}^{\lfloor(k-i) / 2\rfloor}\left(\begin{array}{c}
2 l \\
l
\end{array}\right) \frac{\beta^{l}}{l+1} b_{0}(n-2 l-1, k-l-1) & \text { if } 1 \leq k \leq n, \\
0 & \text { if } k>n .\end{cases}
$$

The proof of Theorem 3.1 is thus completed by replacing (3.33) in (3.6).

Note that in the modified recurrence relation given by $(3.6), b_{i}(n, k)$ is explicitly expressed in terms of $b_{0}(\cdot, \cdot)$ with lower orders of $n$ and $k$ thereby reducing the computational complexity involved in the evaluation of $b_{0}(n, 0)$. 
Proposition 3.4. For all $n \geq 0$,

$$
b_{0}(n, n)= \begin{cases}\frac{1}{k+1}\left(\begin{array}{c}
2 k \\
k
\end{array}\right) \beta^{k} & \text { if } n=2 k \\
0 & \text { if } n=2 k+1 .\end{cases}
$$

Proof. For every $t \geq 0$ and $x \in[0, r t)$, the solution (3.1) can be simplified as

$$
F_{i}(t, x)=\sum_{n=0}^{\infty} e^{-t} \frac{1}{r^{n}} \sum_{k=0}^{n} \frac{x^{k}(r t-x)^{n-k}}{k !(n-k) !} b_{i}(n, k), \quad i=0,1,2, \ldots
$$

Therefore, we get

$$
F_{i}(t, 0)=\lim _{x \rightarrow 0} \sum_{n=0}^{\infty} e^{-t} \frac{t^{n}}{n !} \sum_{k=0}^{n}\left(\begin{array}{l}
n \\
k
\end{array}\right)\left(\frac{x}{r t}\right)^{k}\left(1-\frac{x}{r t}\right)^{n-k} b_{i}(n, k)=\sum_{n=0}^{\infty} e^{-t} \frac{t^{n}}{n !} b_{i}(n, 0) .
$$

Note that $F_{i}(t, 0)=0$ for $i=1,2,3, \ldots$ because when $X(t)=i$, the corresponding net effective rate $r$ is positive. Hence we have

$$
b_{i}(n, 0)=0, \quad n \geq 0, i \geq 1
$$

Also for $t>0$, we have

$$
F_{0}(t, r t)=\lim _{x \rightarrow r t} F_{0}(t, x)=P(X(t)=0)
$$

From (3.35), we obtain

$$
\lim _{x \rightarrow r t} F_{0}(t, x)=\sum_{n=0}^{\infty} e^{-t} \frac{t^{n}}{n !} b_{0}(n, n)
$$

Hence from (3.38) and (3.39), we obtain

$$
\sum_{n=0}^{\infty} e^{-t} \frac{t^{n}}{n !} b_{0}(n, n)=P(X(t)=0) .
$$

Also, from (2.4), we have

$$
P(X(t)=0)=\frac{2 e^{-t} I_{1}(\alpha t)}{\alpha t}=\frac{e^{-t} I_{1}(2 \sqrt{\beta} t)}{\sqrt{\beta} t}=\sum_{k=0}^{\infty} e^{-t} \frac{t^{2 k}}{k !} \frac{\beta^{k}}{(k+1) !} .
$$

Hence, we have

$$
\sum_{n=0}^{\infty} e^{-t} \frac{t^{n}}{n !} b_{0}(n, n)=\sum_{k=0}^{\infty} e^{-t} \frac{t^{2 k}}{k !} \frac{\beta^{k}}{(k+1) !} .
$$

Collecting the coefficients of powers of $t$ on both sides, we get (3.34). 


\section{Fluid queue with chain sequence}

In this way, we determine $b_{0}(n, 0)$ for all $n \geq 1$ from Theorem 3.1 where the $b_{0}(n, k)$ for $0 \leq k \leq n-1$ and hence $b_{1}(n, k)$ for $1 \leq k \leq n$ are obtained from the simple relation established in Proposition 3.2 and Lemma 3.3, respectively. Further, for all $n \geq 1, b_{0}(n, n)$ are calculated from the compact formula derived in Proposition 3.4.

\section{Numerical illustration}

In this section, we discuss the numerical investigation carried out to study the behaviour of $q_{0}(t)$ given by (3.4) with respect to time. Towards this end, we define the truncation step as

$$
N(\varepsilon, t)=\min \left\{n \geq 0 \mid \sum_{h=0}^{n} e^{-t} \frac{t^{h}}{h !} \geq 1-\varepsilon\right\} .
$$

It is easy to check that $N(\varepsilon, t)$ is an increasing function of $t$. So if $q_{0}(t)$ has to be evaluated at $M$ points, say $t_{1}<\cdots<t_{M}$, we only need to evaluate $b_{0}(n, 0)$ for $n=0,1, \ldots$, $N\left(\varepsilon, t_{M}\right)$, and to compute

$$
q_{0}^{(N)}(t)=\sum_{n=0}^{N\left(\varepsilon, t_{M}\right)} e^{-t} \frac{t^{n}}{n !} b_{0}(n, 0) .
$$

Indeed, for every $t \leq t_{M}$, we have

$$
\begin{aligned}
q_{0}(t)-q_{0}^{(N)}(t) & =\sum_{n=N\left(\varepsilon, t_{M}\right)+1}^{\infty} e^{-t} \frac{t^{n}}{n !} b_{0}(n, 0) \\
& \leq \sum_{n=N\left(\varepsilon, t_{M}\right)+1}^{\infty} e^{-t} \frac{t^{n}}{n !}=1-\sum_{n=0}^{N\left(\varepsilon, t_{M}\right)} e^{-t} \frac{t^{n}}{n !}=1-\sum_{n=0}^{N(\varepsilon, t)} e^{-t} \frac{t^{n}}{n !} \leq \varepsilon,
\end{aligned}
$$

where the first inequality comes from the fact that the $b_{i}(n, k)$ are between 0 and 1 as shown in [6].

Below we give the algorithm which we developed to bring out the variations in the form of graphs.

Algorithm 4.1.

input: $t_{1}<\cdots<t_{M}, \varepsilon$

output: $q_{0}^{(N)}\left(t_{1}\right)<\cdots<q_{0}^{(N)}\left(t_{M}\right)$

Compute $N=N\left(\varepsilon, t_{M}\right)$ from relation (4.1).

$b_{0}(0,0)=1 ; b_{1}(0,0)=0$

for $n=1$ to $N$ do

Compute $b_{0}(n, n)$ from relation (3.34).

for $k=n-1$ step -1 to 0 do

Compute $b_{0}(n, k)$ from relation (3.2).

endfor 

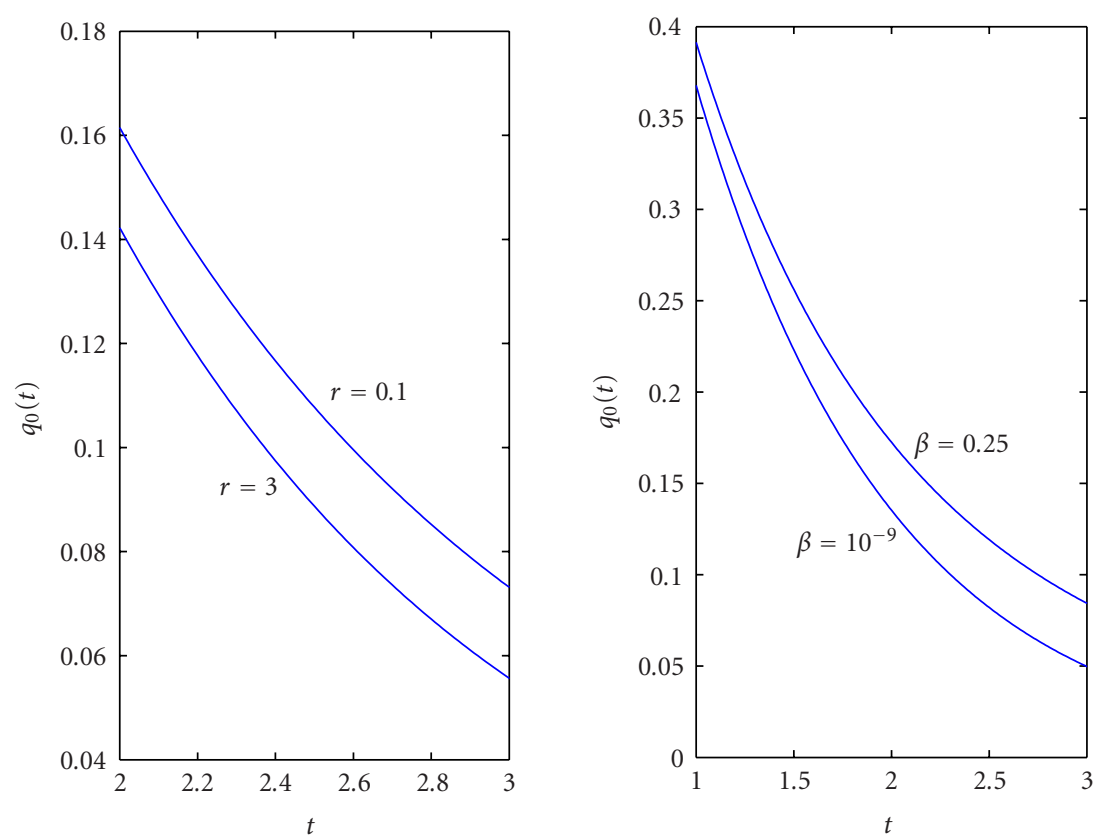

Figure 4.1. Variation of $q_{0}(t)$ against $t$ for varying $r$ when $\beta=0.1$ and varying $\beta$ when $r=1$ with $r_{0}=-1$.

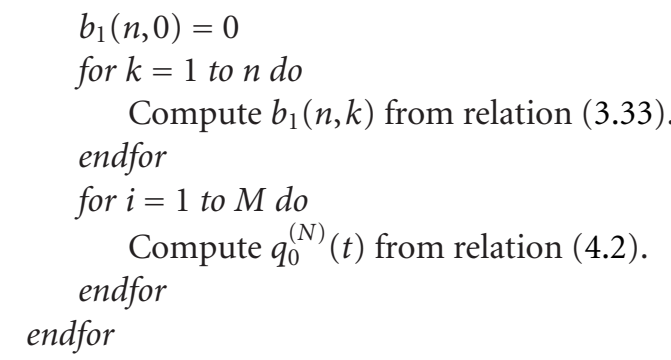

Figure 4.1 depicts the variation of the function $q_{0}(t)$ against $t$ for $r_{0}=-1$, varying values of $r$ when $\beta=0.1$, and varying values of $\beta$ when $r=1$. As the net input rate of the fluid increases, the probability with which the buffer becomes empty over a period of time decreases faster and hence $q_{0}(t)$ approaches zero, as seen in Figure 4.1. In Figure 4.2, the variation in $q_{0}(t)$ against time is plotted for $r=1.0, \beta=0.1$ and $r=0.1, \beta=0.25$. It is observed that for other values of $r$ and $\beta$, the curves lie in the intermediate region.

\section{Analytical solution}

In this section, we present an explicit transient solution for the fluid model under consideration. We discuss the method of continued fractions to solve the governing system 
154 Fluid queue with chain sequence

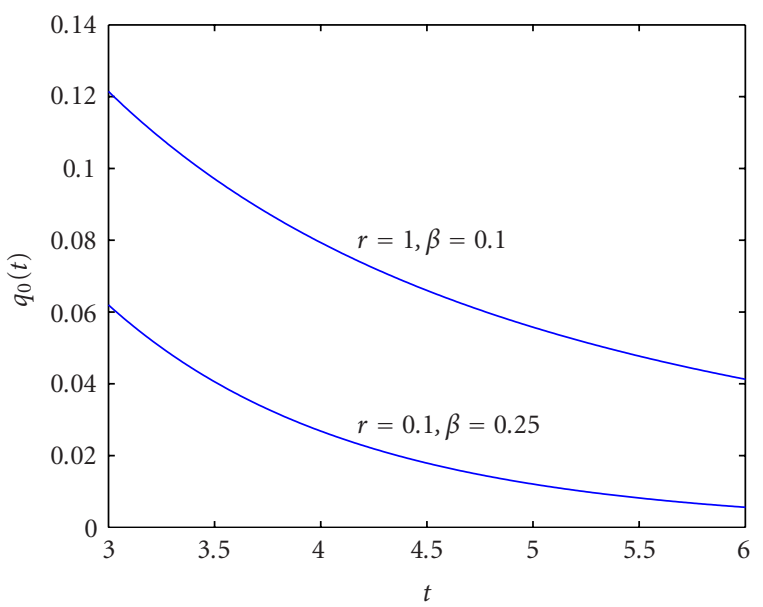

Figure 4.2. Variation of $q_{0}(t)$ against $t$ for varying $r$ and $\beta$ with $r_{0}=-1$.

of partial differential equations. Laplace transform of (2.5) with respect to $t$ yields

$$
\begin{aligned}
& s F_{0}^{*}(s, x)-F_{0}(0, x)+r_{0} \frac{\partial F_{0}^{*}}{\partial x}(s, x)=-F_{0}^{*}(s, x)+\mu_{1} F_{1}^{*}(s, x), \\
& \quad s F_{j}^{*}(s, x)-F_{j}(0, x)+r \frac{\partial F_{j}^{*}}{\partial x}(s, x) \\
& \quad=\lambda_{j-1} F_{j-1}^{*}(s, x)-F_{j}^{*}(s, x)+\mu_{j+1} F_{j+1}^{*}(s, x) \quad \text { for } j=1,2,3, \ldots
\end{aligned}
$$

Taking Laplace transform of (5.1) again with respect to $x$, we get

$$
\begin{gathered}
s F_{0}^{* *}(s, w)-\frac{1}{w}+r_{0} w F_{0}^{* *}(s, w)-r_{0} F_{0}^{*}(s, 0)=-F_{0}^{* *}(s, w)+\mu_{1} F_{1}^{* *}(s, w), \\
s F_{j}^{* *}(s, w)+r w F_{j}^{* *}(s, w)-r F_{j}^{*}(s, 0)=\lambda_{j-1} F_{j-1}^{* *}(s, w)-F_{j}^{* *}(s, w)+\mu_{j+1} F_{j+1}^{* *}(s, w) .
\end{gathered}
$$

Rewriting the above system of equations,

$$
\begin{aligned}
& \left(s+r_{0} w+1\right) F_{0}^{* *}(s, w)-\mu_{1} F_{1}^{* *}(s, w) \\
& \quad=\frac{1}{w}+r_{0} q_{0}^{*}(s)-\lambda_{j-1} F_{j-1}^{* *}(s, w)+(s+1+r w) F_{j}^{* *}(s, w)-\mu_{j+1} F_{j+1}^{* *}(s, w)=0, \quad j \geq 1 .
\end{aligned}
$$

Define

$$
\begin{gathered}
f_{0}^{* *}(s, w)=F_{0}^{* *}(s, w), \\
f_{j}^{* *}(s, w)=(-1)^{j} \frac{\mu_{1} \mu_{2} \cdots \mu_{j}}{r_{0} r^{j-1}} F_{j}^{* *}(s, w) \quad \text { for } j \geq 1 .
\end{gathered}
$$


Making use of the above transformation, (5.3) becomes

$$
\begin{gathered}
\left(w+\frac{s+1}{r_{0}}\right) f_{0}^{* *}(s, w)-f_{1}^{* *}(s, w)=\frac{1}{r_{0} w}+q_{0}^{*}(s), \\
\frac{\lambda_{0} \mu_{1}}{r_{0} r} f_{0}^{* *}(s, w)+\left(w+\frac{s+1}{r}\right) f_{1}^{* *}(s, w)+f_{2}^{* *}(s, w)=0, \\
\frac{\lambda_{j-1} \mu_{j}}{r^{2}} f_{j-1}^{* *}(s, w)+\left(w+\frac{s+1}{r}\right) f_{j}^{* *}(s, w)+f_{j+1}^{* *}(s, w)=0, \quad j=2,3, \ldots
\end{gathered}
$$

These equations can be conveniently rewritten in the form of continued fractions as follows:

$$
\begin{aligned}
& f_{0}^{* *}(s, w)=\frac{1 / r_{0} w+q_{0}^{*}(s)}{w+(s+1) / r_{0}-f_{1}^{* *}(s, w) / f_{0}^{* *}(s, w)}, \\
& \frac{f_{1}^{* *}(s, w)}{f_{0}^{* *}(s, w)}=-\frac{\beta / r_{0} r}{w+(s+1) / r+f_{2}^{* *}(s, w) / f_{1}^{* *}(s, w)}, \\
& \frac{f_{j}^{* *}(s, w)}{f_{j-1}^{* *}(s, w)}=-\frac{\beta / r^{2}}{w+(s+1) / r+f_{j+1}^{* *}(s, w) / f_{j}^{* *}(s, w)} \quad \text { for } j=2,3, \ldots
\end{aligned}
$$

Hence we have

$$
f_{0}^{* *}(s, w)=\frac{1 / r_{0} w+q_{0}^{*}(s)}{w+(s+1) / r_{0}-} \frac{\beta / r_{0} r}{w+(s+1) / r-w+(s+1) / r-} \cdots
$$

Define

$$
\begin{aligned}
f(s, w) & =\frac{1}{w+(s+1) / r-} \frac{\beta / r^{2}}{w+(s+1) / r-}-\frac{\beta / r^{2}}{w+(s+1) / r-} \cdots \\
& =\frac{1}{w+(s+1) / r-\left(\beta / r^{2}\right) f(s, w)} .
\end{aligned}
$$

That is,

$$
\frac{\beta}{r^{2}}(f(s, w))^{2}-\left(w+\frac{s+1}{r}\right) f(s, w)+1=0 .
$$

Solving the above quadratic equation, we obtain

$$
f(s, w)=\frac{(w+(s+1) / r)-\sqrt{(w+(s+1) / r)^{2}-4 \beta / r^{2}}}{2 \beta / r^{2}} .
$$

Using the above definition, we have

$$
\begin{aligned}
& \frac{f_{1}^{* *}(s, w)}{f_{0}^{* *}(s, w)}=-\frac{\beta}{r_{0} r} f(s, w), \\
& \frac{f_{j}^{* *}(s, w)}{f_{j-1}^{* *}(s, w)}=-\frac{\beta}{r^{2}} f(s, w) \quad \text { for } j=2,3 \ldots,
\end{aligned}
$$


156 Fluid queue with chain sequence

and hence

$$
\begin{aligned}
f_{0}^{* *}(s, w) & =\frac{1 / r_{0} w+q_{0}^{*}(s)}{w+(s+1) / r_{0}-\left(\beta / r_{0} r\right) f(s, w)} \\
& =\frac{1 / r_{0} w+q_{0}^{*}(s)}{w+(s+1) / r_{0}-\left(r / 2 r_{0}\right)\left[(w+(s+1) / r)-\sqrt{(w+(s+1) / r)^{2}-4 \beta / r^{2}}\right]}
\end{aligned}
$$

We denote $w+(s+1) / r=\theta$ and $2 \sqrt{\beta} / r=\nu$, then

$$
f_{0}^{* *}(s, w)=\frac{1 / r_{0} w+q_{0}^{*}(s)}{\left(w+(s+1) / r_{0}\right)-\left(r / 2 r_{0}\right)\left(\theta-\sqrt{\theta^{2}-v^{2}}\right)}
$$

From (5.4), $F_{0}^{* *}(s, w)=f_{0}^{* *}(s, w)$, and hence we get

$$
F_{0}^{* *}(s, w)=\left(q_{0}^{*}(s)+\frac{1}{r_{0} w}\right) \sum_{k=0}^{\infty}\left(\frac{r}{2 r_{0}}\right)^{k} \frac{\left(\theta-\sqrt{\theta^{2}-v^{2}}\right)^{k}}{\left(w+(s+1) / r_{0}\right)^{k+1}} \quad \text { for }\left|\frac{\beta f(s, w)}{r\left(r_{0} w+s+1\right)}\right|<1 \text {. }
$$

Also from (5.11), we have

$$
f_{j}^{* *}(s, w)=\frac{r}{r_{0}}\left(-\frac{\beta}{r^{2}} f(s, w)\right)^{j} f_{0}^{* *}(s, w) \quad \text { for } j=1,2,3 \ldots
$$

Getting back to the transformation using (5.4), we obtain

$$
\begin{aligned}
F_{j}^{* *}(s, w) & =\frac{r^{j}}{\mu_{1} \mu_{2} \cdots \mu_{j}}\left(\frac{\beta}{r^{2}} f(s, w)\right)^{j} F_{0}^{* *}(s, w) \\
& =\left(\frac{r}{2}\right)^{j} \frac{1}{\mu_{1} \mu_{2} \ldots \mu_{j}}\left(q_{0}^{*}(s)+\frac{1}{r_{0} w}\right) \sum_{k=0}^{\infty}\left(\frac{r}{2 r_{0}}\right)^{k} \frac{\left(\theta-\sqrt{\theta^{2}-v^{2}}\right)^{j+k}}{\left(w+(s+1) / r_{0}\right)^{k+1}}
\end{aligned}
$$

Hence from (2.3) we have

$$
F_{j}^{* *}(s, w)=\left(\frac{r}{2 \sqrt{\beta}}\right)^{j} U_{j}\left(\frac{1}{2 \sqrt{\beta}}\right)\left(q_{0}^{*}(s)+\frac{1}{r_{0} w}\right) \sum_{k=0}^{\infty}\left(\frac{r}{2 r_{0}}\right)^{k} \frac{\left(\theta-\sqrt{\theta^{2}-v^{2}}\right)^{j+k}}{\left(w+(s+1) / r_{0}\right)^{k+1}} .
$$

Inverting the above expression, we obtain the transient buffer content distribution as given in the following theorem. 
Theorem 5.1. For every $t \geq 0$ and $x \in[0, r t)$, we have

$$
\begin{aligned}
& F_{0}(t, x)=e^{-x / r_{0}} q_{0}\left(t-\frac{x}{r_{0}}\right)+e^{-t}-e^{-x / r_{0}} e^{-\left(t-x / r_{0}\right)} \\
& +\sum_{k=1}^{\infty}\left(\frac{r}{2 r_{0}}\right)^{k} \int_{0}^{x} e^{-(1 / r)(x-y)} \frac{\nu^{k} k I_{k}(\nu(x-y))}{k !(x-y)} \\
& \cdot\left\{y^{k} e^{-y / r_{0}} H\left(t-\frac{x-y}{r}-\frac{y}{r_{0}}\right) q_{0}\left(t-\frac{x-y}{r}-\frac{y}{r_{0}}\right)\right. \\
& \left.+r_{0}^{k} e^{-(t-(x-y) / r)}\left(t-\frac{x-y}{r}\right)^{k}\right\} d y, \\
& F_{j}(t, x)=\left(\frac{r}{2 \sqrt{\beta}}\right)^{j} U_{j}\left(\frac{1}{2 \sqrt{\beta}}\right) \\
& \times \sum_{k=0}^{\infty}\left(\frac{r}{2 r_{0}}\right)^{k} \int_{0}^{x} e^{-(1 / r)(x-y)} \frac{\nu^{j+k}(j+k) I_{j+k}(\nu(x-y))}{k !(x-y)} \\
& \cdot\left\{y^{k} e^{-y / r_{0}} H\left(t-\frac{x-y}{r}-\frac{y}{r_{0}}\right) q_{0}\left(t-\frac{x-y}{r}-\frac{y}{r_{0}}\right)\right. \\
& \left.+r_{0}^{k} e^{-\lambda(t-(x-y) / r)}\left(t-\frac{x-y}{r}\right)^{k}\right\} d y \quad \text { for } j=1,2, \ldots,
\end{aligned}
$$

where $H(\cdot)$ denotes the Heaviside function.

\section{Acknowledgment}

P. R. Parthasarathy and K. V. Vijayashree thank Av. Humboldt Foundation, Germany, and National Board of Higher Mathematics (NBHM), India, respectively, for the financial assistance during the preparation of the paper.

\section{References}

[1] I. J. B. F. Adan and J. A. C. Resing, Simple analysis of a fluid queue driven by an $M / M / 1$ queue, Queueing Syst. Theory Appl. 22 (1996), no. 1-2, 171-174.

[2] N. Barbot and B. Sericola, Stationary solution to the fluid queue fed by an $M / M / 1$ queue, J. Appl. Probab. 39 (2002), no. 2, 359-369.

[3] V. G. Kulkarni, Fluid models for single buffer systems, Frontiers in Queueing (J. H. Dshalalow, ed.), Probab. Stochastics Ser., CRC, Florida, 1997, pp. 321-338.

[4] R. B. Lenin and P. R. Parthasarathy, A birth-death process suggested by a chain sequence, Comput. Math. Appl. 40 (2000), no. 2-3, 239-247.

[5] P. R. Parthasarathy and K. V. Vijayashree, Fluid queues driven by a discouraged arrivals queue, Int. J. Math. Math. Sci. 2003 (2003), no. 24, 1509-1528.

[6] Fluid queues driven by birth and death processes with quadratic rates, Int. J. Comput. Math. 80 (2003), no. 11, 1385-1395.

[7] P. R. Parthasarathy, K. V. Vijayashree, and R. B. Lenin, An M/M/1 driven fluid queue-continued fraction approach, Queueing Syst. Theory Appl. 42 (2002), no. 2, 189-199. 
158 Fluid queue with chain sequence

[8] Q. Ren and H. Kobayashi, Transient solutions for the buffer behavior in statistical multiplexing, Performance Evaluation 23 (1995), 65-87.

[9] B. Sericola, Transient analysis of stochastic fluid models, Performance Evaluation 32 (1998), 245263.

[10]_, A finite buffer fluid queue driven by a Markovian queue, Queueing Syst. Theory Appl. 38 (2001), no. 2, 213-220.

[11] A. Simonian and J. Virtamo, Transient and stationary distributions for fluid queues and input processes with a density, SIAM J. Appl. Math. 51 (1991), no. 6, 1732-1739.

[12] T. Tanaka, O. Hashida, and Y. Takahashi, Transient analysis of fluid model for ATM statistical multiplexer, Performance Evaluation 23 (1995), no. 2, 145-162.

[13] E. A. van Doorn and W. R. W. Scheinhardt, A fluid queue driven by an infinite-state birth-death process, Proc. 15th International Teletraffic Congress (Washington, DC) (V. Ramaswami and P. E. Wirth, eds.), Elsevier, Amsterdam, 1997, pp. 465-475.

[14] J. Virtamo and I. Norros, Fluid queue driven by an M/M/1 queue, Queueing Syst. Theory Appl. 16 (1994), no. 3-4, 373-386.

P. R. Parthasarathy: Department of Mathematics, Indian Institute of Technology, Madras, Chennai 600 036, India

E-mail address: prp@iitm.ac.in

B. Sericola: IRISA-INRIA, Campus Universitaire de Beaulieu, 35042 Rennes Cedex, France

E-mail address: bruno.sericola@irisa.fr

K. V. Vijayashree: Department of Mathematics, Indian Institute of Technology, Madras, Chennai 600036 , India

E-mail address: ma99p01@violet.iitm.ernet.in 


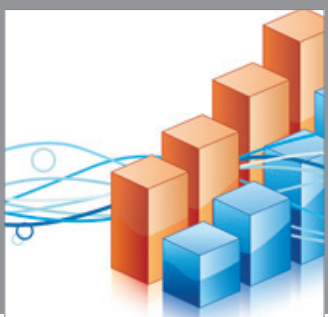

Advances in

Operations Research

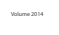

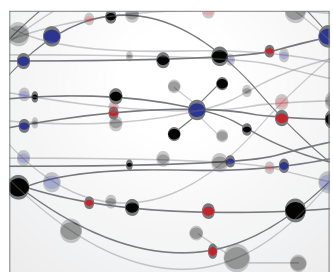

\section{The Scientific} World Journal
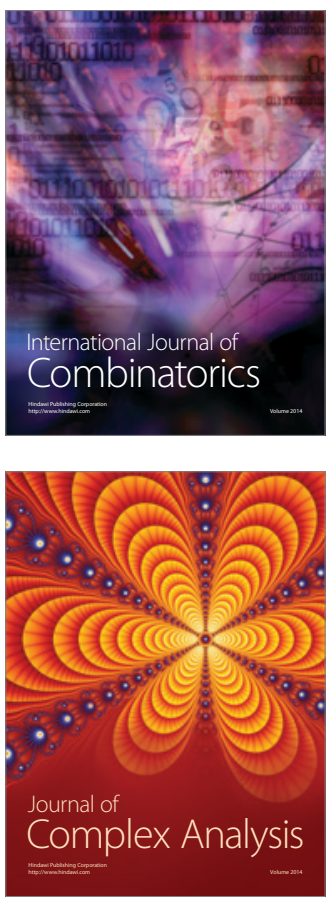

International Journal of

Mathematics and

Mathematical

Sciences
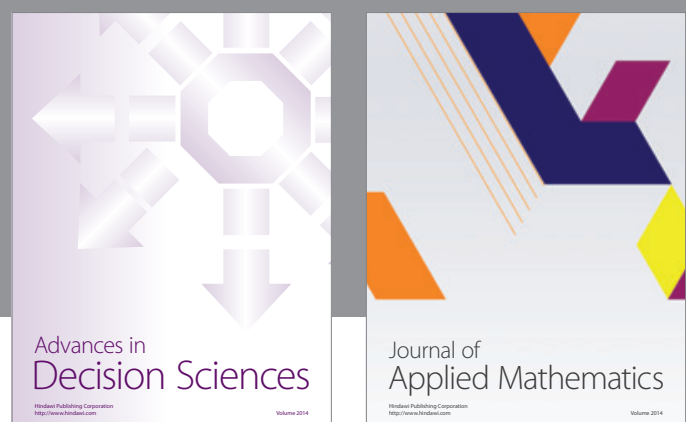

Journal of

Applied Mathematics
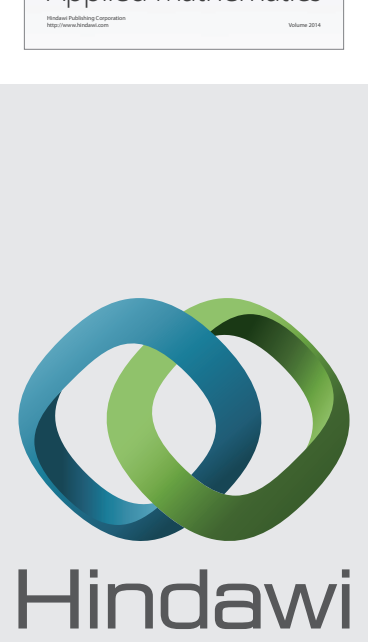

Submit your manuscripts at http://www.hindawi.com
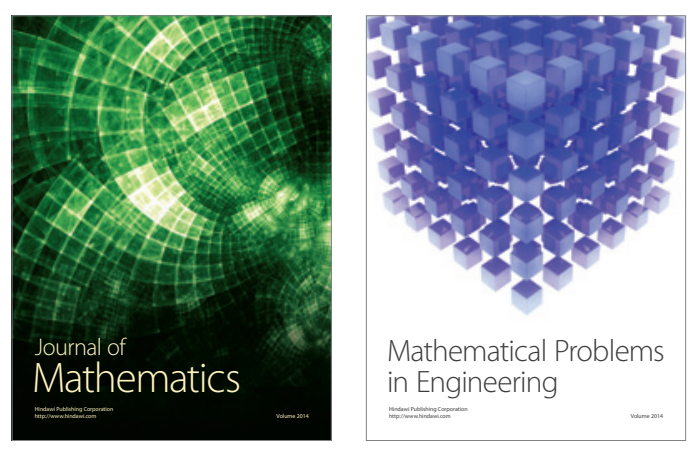

Mathematical Problems in Engineering
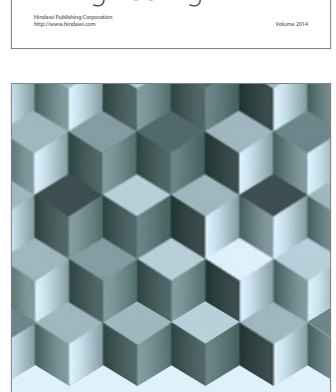

Journal of

Function Spaces
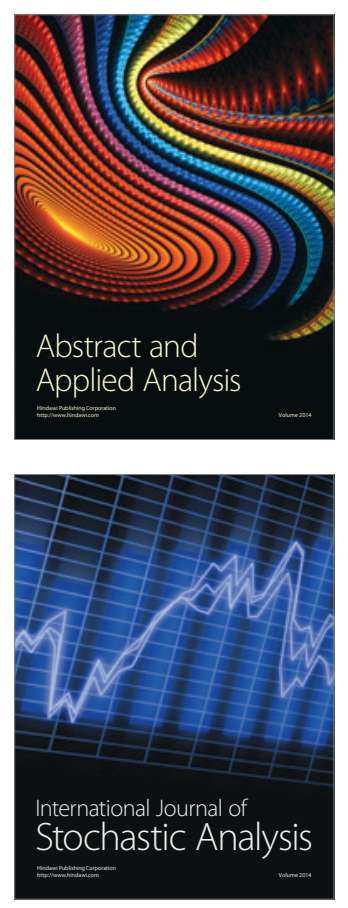

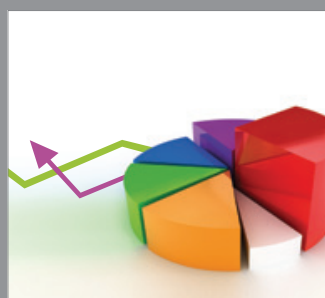

ournal of

Probability and Statistics

Promensencen
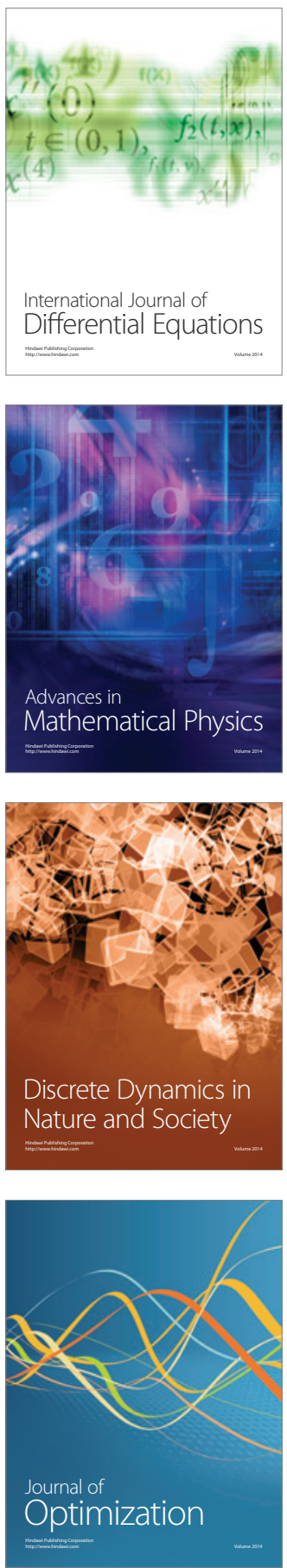